The AAPS Journal 2007; 9 (3) Article 38 (http://www.aapsj.org).

\title{
Corrigendum: 2007 Highlights of Advances in the Pharmaceutical Sciences: An American Association of Pharmaceutical Scientists (AAPS) Perspective
}

Received: August 7, 2007; Accepted: August 7, 2007; Published: September 28, 2007

Marilyn N. Martinez, ${ }_{1}^{1}$ Peter Bonate, ${ }^{2}$ Robert L. Chapman, ${ }^{3}$ Anne De Groot, ${ }^{4}$ Susan D'Souza, ${ }^{5}$ Naushad Ghilazi, ${ }^{6}$ Vivian Gray, ${ }^{7}$ Vishal K. Gupta, ${ }^{8}$ Kim Huynh-Ba, ${ }^{9}$ Sunil Iyer, ${ }^{10}$ Arya Jayatilaka, ${ }^{11}$ Amita Joshi, ${ }^{12}$ H. Thomas Karnes, ${ }^{10}$ Mansoor Khan, ${ }^{1}$ Patrick Liu, ${ }^{13}$ Craig Lunte, ${ }^{14}$ Christopher R. McCurdy, ${ }^{15}$ Marilyn E. Morris, ${ }^{16}$ Kenneth J. Norris, ${ }^{17}$ Phillip Ramsey, ${ }^{18}$ Sanjay Sehgal, ${ }^{19}$ and Manuel Zahn ${ }^{20}$

AAPS Journal. 2007;9(2):E219-E226. DOI: 10.1208/aapsj0902024

The author affiliation information for Susan D'Souza ${ }^{5}$ was incorrect and should have read:

${ }^{5}$ Schering-Plough Research Institute, Summit, NJ 